\title{
Pole versus Breit-Wigner resonance description of the orbitally excited baryons
}

\author{
Ron Workman \\ Department of Physics, Virginia Tech, Blacksburg, Virginia 24061
}

(Received 16 November 1998; revised manuscript received 4 February 1999)

\begin{abstract}
We consider the masses used in recent studies involving the nonstrange sector of the $l=1$ baryons. The use of $T$-matrix and $K$-matrix poles versus the conventional Breit-Wigner masses is discussed within the context of a large- $N_{c}$ fitting scheme. [S0556-2813(99)01206-6]

PACS number(s): 24.85.+p, 12.38.Bx, 14.20.Gk
\end{abstract}

While the mass and charge of a particle are typically the easiest quantities to determine experimentally, the proper way to define and extract the mass of an unstable state continues to be controversial. This issue has been extensively debated in studies of the $Z^{0}$ mass [1] and has been discussed, in the context of baryon resonances, by Höhler [2]. While resonancelike behavior is, in principle, possible without a pole in the $S$ matrix [3], the pole position has many features one would associate with the physical mass. These include $[1,4]$ independence from the production process, factorizability of the residue, and gauge independence. Other possibilities include the bare, $K$-matrix, and Breit-Wigner (BW) masses.

The Particle Data Group (PDG) has until recently listed only the BW masses and widths in its Baryon Summary Tables [5], though pole positions have been added in the most recent edition. Most fits involving either quark model or large- $N_{c}$ formalisms have been carried out using these BW values. However, as emphasized by Höhler [2], the BW values quoted by the PDG are inherently model dependent. This has led us to ask two important questions. (1) Are the above fits influenced by differences between the BW and pole masses? (2) Which definitions of the mass actually correspond to the quantities being calculated?

The first question can be answered most easily. For this purpose, we have examined several fits to resonance properties utilizing the large- $N_{c}$ formalism. In a series of papers, the orbitally excited SU(6) 70-plet baryons have been analyzed in terms of their BW masses [6], and strong [7] and electromagnetic [8] decays, within the framework of large$N_{c}$ QCD. While such fits necessarily involve a large number of free parameters, a comparison of the parameters determined in these independent fits reveals a remarkably consistency. This is particularly evident if one compares the mixing angles associated with, in $\pi N$ notation, the $S_{11}$ and $D_{13}$ resonances. The two $S_{11}$ mass eigenstates $N(1535)$ and $N(1650)$ and two $D_{13}$ mass eigenstates $N(1520)$ and $N(1700)$ are mixtures of states, $N_{i j}$, with total quark spin $i / 2$ and total angular momentum $j / 2$, as parametrized by mixing angles:

$$
\left[\begin{array}{l}
N(1535) \\
N(1650)
\end{array}\right]=\left[\begin{array}{cc}
\cos \theta_{N 1} & \sin \theta_{N 1} \\
-\sin \theta_{N 1} & \cos \theta_{N 1}
\end{array}\right]\left[\begin{array}{c}
N_{11} \\
N_{31}
\end{array}\right]
$$

and
The mixing angles $\theta_{N 1}$ and $\theta_{N 3}$ have been determined independently in Refs. [6-8]. Results for the angles are identical, within the quoted uncertainties, in these fits to the masses and decay widths (both strong and electromagnetic). This self-consistency adds considerable weight to the large- $N_{c}$ fitting scheme.

We have repeated the mass fit of Ref. [6] using instead a set of pole masses [9-11], where the mass was taken to to be the real part of the pole position. For these resonances, the difference in definition is quite important. This is apparent if one notes that the heaviest and lightest of the orbitally excited SU(6) 70-plet baryons are separated by only about 200 $\mathrm{MeV}$, whereas the difference between BW and pole masses can be $50 \mathrm{MeV}$ or more.

While any comparison necessarily depends upon the number of operator coefficients varied in the fit, an interesting result follows if one fits the seven masses with six parameters and predicts the two mixing angles. These parameters scale the $\mathrm{O}\left(N_{c}\right)$ and $\mathrm{O}(1)$ contributions and the largest terms of $\mathrm{O}\left(N_{c}^{-1}\right)$. A detailed description of this method and a complete set of relations between the parameters and masses are given in Ref. [6]. In the present Brief Report, we retain this notation [6] in order to aid comparison. The six-parameter fit of Ref. [6] was able to successfully reproduce the BW masses and mixing angles in agreement with the results of Refs. $[7,8]$. A nine-parameter fit, including the mixing angles as data, did not give qualitatively different results [6]. (The values given in Ref. [6] were first verified before considering the effect of pole masses [12].) Our fits, using both BW and pole masses, and the resulting parameters and mixing angles are given in Tables I and II.

While the mixing angles resulting from the pole fit are quite different from those found in Refs. [6-8], the other parameters display a number of similarities. The relative signs have not changed and the coefficient $c_{2}$ remains small and consistent with zero. Apart from $c_{2}$, the coefficients found in the pole-mass fit are of the same magnitude. The other terms of $\mathrm{O}\left(N_{c}^{-1}\right)$ listed and considered in Ref. [6] appear to be unimportant in both the BW and pole-mass fits. It is also interesting to see that, using either set of masses, a significant part of the overall chi squared is due to the $N(1700)$. This state has a very weak coupling to the $\pi N$ 
TABLE I. Six-parameter fit to BW masses. The predicted mixing angles are $\theta_{N 1}=0.53 \mathrm{rad}$ and $\theta_{N 3}=3.06 \mathrm{rad}$. (Values from Ref. [7] are $\theta_{N 1}=0.61 \pm 0.09 \mathrm{rad}$ and $\theta_{N 3}=3.04 \pm 0.15 \mathrm{rad}$.) Here $\chi^{2} / N_{\mathrm{DF}}=0.23$. Mixing angles were not included as data.

\begin{tabular}{lccc}
\hline \hline & Fit $(\mathrm{MeV})$ & Expt. (MeV) & Parameters $(\mathrm{MeV})$ \\
\hline$\Delta(1700)$ & 1712 & $1720 \pm 50$ & $c_{1}: 466 \pm 14$ \\
$\Delta(1620)$ & 1643 & $1645 \pm 30$ & $c_{2}:-29.5 \pm 39$ \\
$N(1675)$ & 1678 & $1678 \pm 8$ & $c_{3}: 303 \pm 141$ \\
$N(1700)$ & 1712 & $1700 \pm 50$ & $c_{4}: 69 \pm 99$ \\
$N(1650)$ & 1660 & $1660 \pm 20$ & $c_{5}: 63 \pm 46$ \\
$N(1520)$ & 1523 & $1523 \pm 8$ & $c_{6}: 424 \pm 86$ \\
$N(1535)$ & 1539 & $1538 \pm 18$ & \\
\hline \hline
\end{tabular}

channel, and has not been detected in all analyses of elastic $\pi N$ scattering data. As a result, its mass and pole position are not well determined.

In an expanded version of Ref. [6], the relative sizes of the fitted coefficients have been used to suggest that the underlying dynamics is due to effective pseudoscalar-meson exchanges among the quarks [13]. As the pole mass fit chooses a different set of dominant coefficients, we see that differences in definition are important. Thus we are forced to consider the second (much harder) question: Which "mass", is most appropriate?

We first assume that the pole positions are eigenvalues of an operator $M-(i / 2) \Gamma$ in the sense that

$$
\left(M-\frac{i}{2} \Gamma\right)|A\rangle=m_{A}|A\rangle,
$$

where $m_{A}$ is complex. The connection with most model approaches is a neglect of $\Gamma$, resulting in real mass values. For the states under consideration, terms of order $N_{c}, 1$, and $N_{c}^{-1}$ have been included in fits to the masses. As the widths are expected to enter at $\mathrm{O}\left(N_{c}^{-2}\right)[14]$, the neglect of $\Gamma$ appears to be completely consistent.

In order to more closely examine the mixed states $N(1535)$ and $N(1650)$ or $N(1520)$ and $N(1700)$, we follow an argument given by Aitchison [15] for overlapping resonances. In this case, $M-(i / 2) \Gamma$ is an effective Hamiltonian matrix. In terms of states, denoted by Greek indices, which diagonalize $M$ (but not the full Hamiltonian), the $T$ matrix has the form [15]

TABLE II. Six-parameter fit to pole masses. The predicted mixing angles are $\theta_{N 1}=2.63 \mathrm{rad}$ and $\theta_{N 3}=0.35 \mathrm{rad}$. Here $\chi^{2} / N_{\mathrm{DF}}$ $=0.005$. Mixing angles were not included as data.

\begin{tabular}{lccc}
\hline \hline & Fit $(\mathrm{MeV})$ & Expt. $(\mathrm{MeV})$ & Parameters $(\mathrm{MeV})$ \\
\hline$\Delta(1700)$ & 1655 & $1655 \pm 10$ & $c_{1}: 497 \pm 13$ \\
$\Delta(1620)$ & 1585 & $1585 \pm 15$ & $c_{2}:-1.7 \pm 18$ \\
$N(1675)$ & 1660 & $1660 \pm 10$ & $c_{3}: 196 \pm 87$ \\
$N(1700)$ & 1647 & $1650 \pm 50$ & $c_{4}: 186 \pm 26$ \\
$N(1650)$ & 1670 & $1670 \pm 20$ & $c_{5}: 104 \pm 21$ \\
$N(1520)$ & 1510 & $1510 \pm 5$ & $c_{6}: 212 \pm 70$ \\
$N(1535)$ & 1510 & $1510 \pm 10$ & \\
\hline \hline
\end{tabular}

$$
T_{i j}=f_{i \alpha} S_{\alpha \beta}^{\prime} f_{\beta j}
$$

where

$$
S_{\alpha \beta}^{\prime-1}=S_{\alpha \beta}^{-1}-\Sigma_{\alpha \beta}
$$

and

$$
S_{\alpha \beta}^{-1}=\left(m_{\alpha}-m\right) \delta_{\alpha \beta}, \quad \Sigma_{\alpha \beta}=\frac{i}{2} \sum_{i} 2 \pi \rho_{i} f_{\alpha i} f_{i \beta} .
$$

In the above, $m$ is the energy, $f_{\alpha i}$ is the coupling between the resonant state $\alpha$ and the continuum state $i$, and $\rho_{i}$ is the phase space factor for channel $i$. The $T$ matrix can then be written, in terms of the $K$ matrix, as $T=K(1-i \pi \rho K)^{-1}$, wherein the $K$ matrix has the form

$$
K_{i j}=f_{i \alpha} S_{\alpha \beta} f_{\beta j}
$$

The neglect of $\Gamma$ results in the approximation $T_{i j} \simeq K_{i j}$. Therefore, as one might expect, $K$ - and $T$-matrix poles are equivalent in the absence of a width.

At this point a few comments are necessary. First, it is known that the $K$ - and $T$-matrix masses are separated by amounts similar to the difference between the BW and $T$-matrix pole masses $[11,16]$. Thus, one or both of the $K$ and $T$-matrix masses must shift significantly in the presence of a width. From the fits in Tables I and II, we see that the masses can be reproduced, to the few $\mathrm{MeV}$ level, without $\mathrm{O}\left(N_{c}^{-2}\right)$ terms. As a result, we expect the $K$-matrix mass to remain relatively stable. However, in Eq. (3) we see that a width alters both the effective Hamiltonian and the basis states. As this width is not small, being typically $150 \mathrm{MeV}$, a moderate shift in the real part of $m_{A}$ should be expected. In matching phenomenological masses from data fits to the formalism of Refs. $[6,13]$, we require a quantity which remains stable as the width is turned on. As a result we suggest that the (real) $K$-matrix poles are most closely associated with the large- $N_{c}$ formalism of Refs. [6-8,13]. While $K$-matrix pole positions are not tabulated by the PDG, a recent study [11] finds that, at least for the $N(1535)$, the $\mathrm{BW}$ and $K$-matrix masses are in reasonable agreement.

In summary, after comparing the various definitions used to extract masses from experimental data, at least for the considered set of resonances, we find the $K$-matrix definition to be most appropriate when comparing with large- $N_{c}$ results. One might object that large- $N_{c}$ QCD should be giving the more physical $T$-matrix result. This is not a problem, as the above argument implies that a comparison of phenomenological $K$-matrix masses is essentially equivalent to a comparison of $T$-matrix masses to $\mathrm{O}\left(N_{c}^{-1}\right)$. One final point should be emphasized. In this study, we have completely ignored the effects of nonresonant background contributions. This would not have been a problem had the $T$-matrix pole been favored. However, the $K$-matrix pole is influenced by background contributions, and thus a degree of model dependence appears unavoidable [17]. As a final point, we mention that some recent studies [18] have found photodecay ampli- 
tudes for the $N(1535)$ and $N(1520)$ which strongly contradict the PDG values fitted in Ref. [8]. In the author's opinion, it would be extremely useful to determine whether these results preserve the consistent picture found in Refs. $[6-8,13]$.
We thank Carl Carlson and Chris Carone for many helpful discussions. A useful communication from H.B. O'Connell is also acknowledged. This work was supported in part by U.S. Department of Energy Grant No. DE-FG0297ER41038.
[1] See for example B.A. Kniehl and A. Sirlin, Phys. Rev. Lett. 81, 1373 (1998); R.G. Stuart, ibid. 70, 3193 (1993).

[2] G. Höhler, $\pi N$ Newslett. 10, 320 (1997); 14, 168 (1998), and references therein.

[3] L. Fonda, G.C. Ghirardi, and A. Rimini, Rep. Prog. Phys. 41, 587 (1978).

[4] M. Lévy, Nuovo Cimento 13, 115 (1959); H.P. Stapp, ibid. 32, 103 (1964). For a more recent work, see M. Benayoun, H.B. O'Connell, and A.G. Williams, Phys. Rev. D 59, 074020 (1999).

[5] R.M. Barnett et al., Phys. Rev. D 54, 1 (1996); C. Caso et al., Eur. Phys. J. C 3, 1 (1998).

[6] C.E. Carlson, C.D. Carone, J.L. Goity, and R.F. Lebed, Phys. Lett. B 438, 327 (1998).

[7] C.D. Carone, H. Georgi, L. Kaplan, and D. Morin, Phys. Rev. D 50, 5793 (1994).

[8] C.E. Carlson and C.D. Carone, Phys. Rev. D 58, 053005 (1998).

[9] The pole masses fitted in Table II were estimated from the results of Refs. $[5,10,11]$. The $N(1700)$ state was not detected in the analysis of Ref. [10].

[10] R.A. Arndt, I.I. Strakovksy, R.L. Workman, and M.M. Pavan, Phys. Rev. C (submitted).
[11] A.M. Green and S. Wycech, Phys. Rev. C 55, R2167 (1997); R.A. Arndt, A.M. Green, R.L. Workman, and S. Wycech, ibid. 58, 3636 (1998).

[12] The tables of coefficients and chi-squared values were reproduced after many invaluable communications with the authors of Ref. [6].

[13] C.E. Carlson, C.D. Carone, J.L. Goity, and R.F. Lebed, Report No. JLAB-THY-98-50, hep-ph/9812440.

[14] A. Wirzba, M. Kirchbach, and D.O. Riska, J. Phys. G 20, 1583 (1994).

[15] I.J.R. Aitchison, Nucl. Phys. A189, 417 (1972).

[16] For more detailed comparisons of BW, $K$-matrix, and $T$-matrix fits, see, for example, R.S. Longacre, T. Lasinski, A.H. Rosenfeld, G. Smadja, R.J. Cashmore, and D.W.G.S. Leith, Phys. Rev. D 17, 1795 (1978).

[17] A number of relations between the $T$-matrix and $K$-matrix formalisms are given in R. Levi Setti and T. Lasinski, Strongly Interacting Particles (University of Chicago Press, Chicago, 1973).

[18] B. Krusche, N.C. Mukhopadhyay, J.-F. Zhang, and M. Benmerrouche, Phys. Lett. B 397, 171 (1997); B. Krusche, Phys. Rev. Lett. 74, 3736 (1995); N.C. Mukhopadhyay and N. Mathur, Phys. Lett. B 444, 7 (1998). 